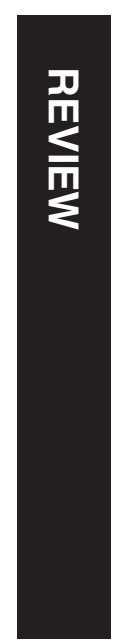

\title{
Fingolimod: therapeutic mechanisms and ocular adverse effects
}

\begin{abstract}
Fingolimod is an oral immunomodulating drug used in the management of relapsing-remitting multiple sclerosis (RRMS). We aim to review the published literature on ocular manifestations of fingolimod therapy and their possible underlying mechanisms. The therapeutic effects of fingolimod are mediated via sphingosine receptors, which are found ubiquitously in various organs, including lymphoid cells, central nervous system, cardiac myocytes, and smooth muscle cells. Fingolimod-associated macular oedema (FAME) is the most common ocular side effect but retinal haemorrhages and retinal vein occlusion can occur. The visual consequences appear to be mild and, in cases of FAME, resolution is often attained with discontinuation of therapy. However, in cases of retinal vein occlusion, discontinuation of fingolimod alone may not be sufficient and intra-vitreal therapy may be required. We also propose a pragmatic service pathway for monitoring patients on fingolimod therapy, which includes stratifying them by risk and visual acuity.

Eye (2017) 31, 232-240; doi:10.1038/eye.2016.258; published online 25 November 2016
\end{abstract}

${ }^{4}$ School of Life \& Health Sciences, Aston University, Birmingham, UK

Correspondence: Y Yang, Department of Ophthalmology, The Royal Wolverhampton NHS Trust, New Cross Hospital, Wednesfield Road, Wolverhampton WV10 OQP, UK

Tel: +44 (0)19 0230 7999;

Fax: +44 (0)1902695600.

E-mail: yit.yang@nhs.net

${ }^{5}$ Joint first author.

Received: 10 May 2016

Accepted: 2 October 2016

Published online:

25 November 2016

\section{Introduction}

Fingolimod is the first orally administered agent to be licensed by the Food and Drug Administration (FDA), and also approved by the National Institute for Health and Care Excellence (NICE), for use in patients with highly active relapsing remitting multiple sclerosis (RRMS) after recent clinical trials demonstrated its efficacy in reducing the frequency of relapses and disability progression on long-term follow-up of patients with multiple sclerosis when compared with placebo. ${ }^{1,2}$ These therapeutic effects in multiple sclerosis therapy are thought to be due to the action of fingolimod on preventing the egression of lymphocytes
P Mandal' ${ }^{1}$, A Gupta ${ }^{1,5}$, W Fusi-Rubiano ${ }^{1}$, PA Keane ${ }^{2,3}$ and $Y$ Yang ${ }^{1,4}$ from lymphoid tissue into the circulation, thereby sparing the central nervous system from attack by myelin-reactive lymphocytes. ${ }^{3,4}$ In vivo, fingolimod exerts this immunomodulating effect through a novel mechanism by binding sphingosine-1-phosphate (S1P) receptors on lymphocytes. Although S1P receptors are found with the highest density in leucocytes and lymphoid tissue, they are also widely expressed in many cell types in other organs systems, including the heart, brain, liver, stomach, and probably also in the retina. This ubiquitous nature of the target receptor for fingolimod accounts for the wide range of adverse effects, including hypertension, heart block,

bradycardia, and macular oedema.

In the original pivotal FREEDOMS study, all seven patients with macular oedema had been randomised to, and received, fingolimod. ${ }^{1}$ Since its launch in 2010, numerous reports of a variety of adverse events associated with fingolimod therapy have been published. This has led to the recommendation that patients should have cardiac and ophthalmic evaluation prior to commencing fingolimod and at every 3-4 months during therapy. ${ }^{5}$

Given the recent anecdotal reports of
Fingolimod-Associated Macular oEdema (FAME) and other retinal complications, coupled with the paucity of the data on the putative pathogenic mechanisms responsible for the effect of fingolimod in the eye, we have reviewed the relevant published literature with the aim of summarising the current concepts on the mode of action of fingolimod, collating the available body of clinical experience on diagnosis, treatment and outcomes of ophthalmic complications presumed to be associated with fingolimod therapy. This article should provide ophthalmologists with an initial patients on fingolimod therapy in clinical practice and should also provide some insight current reference base for the management of 
into the possible pathogenic mechanisms responsible, which could serve to focus our thoughts on the development of more specifically targeted therapy for these novel retinal problems. With this information, we then go on to suggest a pragmatic care pathway for the ophthalmic monitoring of patients on fingolimod therapy.

\section{Fingolimod's mode of action and its cardiovascular side effects}

In vivo, fingolimod is phosphorylated to fingolimodphosphate and becomes structurally similar to a sphingolipid called sphingosine-1-phosphate (S1P), an extracellular mediator, preventing it from binding normally to the five types of S1P receptors $\left(\mathrm{S}_{\mathrm{P}} \mathrm{PR}_{1-5}\right)$. At the cellular level, it leads to internalisation and eventual degradation of these cell surface receptors and abnormal cellular function and communication. 4,6 The S1P receptors are found on lymphocytes and other organs, and whilst in MS, it has the desired therapeutic effect of reducing the upregulation of lymphocytes and their migration from lymphoid tissue into the circulation and the central nervous system, the destruction of S1P receptors in other organs is responsible for its cardiovascular and probably also retinal side effects.

The location of S1P receptors on cardiac myocytes and smooth muscle cells is probably responsible for the adverse cardiovascular effects of fingolimod, which include bradycardia, atrioventricular nodal block, and systemic hypertension. Bradycardia occurs in $0.6 \%$ of patients treated with fingolimod. It is typically observed 4-5 $\mathrm{h}$ after the first dose, with a mean maximum heart rate reduction of $8 \mathrm{bpm}$ (beats per minute). The $\mathrm{S}_{\mathrm{PPR}}$ activation with fingolimod activates G-protein coupled inwardly-rectifying potassium channels (GIRKs) on myocytes. This leads to an efflux of potassium, thereby hyperpolarising the cell membrane and temporarily reducing excitability. This effect is transient but leads to internalisation of $S 1 P R_{1}$. This same mechanism is responsible for Mobitz type 1 s-degree AV nodal block observed in $0.2 \%$ of those patients treated with fingolimod. ${ }^{6,7}$ An increase in blood pressure is observed in patients treated with fingolimod. This increase is, on average, $+2 \mathrm{~mm} \mathrm{Hg}$ systolic and $+1 \mathrm{~mm} \mathrm{Hg}$ diastolic. This effect is believed to be via the presence of $S 1 \mathrm{PR}_{1}, \mathrm{~S}_{\mathrm{PR}}$, and $\mathrm{S}_{\mathrm{PRR}}$ receptors in arterial smooth muscle cells. Activation of $\mathrm{S}_{\mathrm{PR}}$ in arterial smooth muscle cells causes increased nitric oxide production (therefore vasodilation) as well as an intracellular increase in calcium. This rise in calcium causes an increase in smooth muscle contraction; therefore these opposing effects initially offset one another. However, once $\mathrm{S}_{\mathrm{PR}}$ internalises, binding shifts to $\mathrm{S}_{\mathrm{PR}}$ and $\mathrm{S} 1 \mathrm{PR}_{3}$ that are also found on arterial smooth muscle cells, thus smooth muscle contraction is the over- riding force. This effect on blood pressure is prolonged longer than first-dose related bradycardia and AV nodal block, with a peak at 6 months after which it stabilises. ${ }^{6}$

\section{Fingolimod and the eye}

In the retina, the effects of fingolimod on the actions of the sphingolipid, sphingosine-1-phosphate, and its S1P receptors are less well-understood. Sphingolipids are the third most abundant lipid in the retina. ${ }^{8}$ It is well recognised that sphingolipid metabolism have important roles in retinal cell death and survival. This balance is referred to as the 'sphingolipid rheostat.' Ceramide (Cer) is the key metabolite for sphingolipid production. There are two major pathways for Cer production-de novo synthesis for higher-order sphingolipids and the recycling/degradation of higher order sphingolipids. Aberrant sphingolipid metabolism is known to cause various metabolic storage diseases such as Tay-Sachs, Fabry's disease, and Niemann-Pick disease. ${ }^{9}$ Although fingolimod can potentially affect sphingolipid metabolism globally in the whole retina by inhibiting Cer enzymes, thereby reducing the formation of de novo Cer, ${ }^{9}$ it seems that this is unlikely to be pathogenic mechanism behind the causation of macular oedema that have been reported in the recent literature as all the cases have reported very localised distribution of oedema to the macular area only and not globally across the whole retina. ${ }^{10-21}$

There are a number of ocular conditions that have been linked to fingolimod. The most common is fingolimodassociated macular oedema, the only ocular condition to have been mentioned in the original FREEDOMS and TRANSFORMS trials, as well as in the drug marketing literature. However, since 2010, when fingolimod has been used outside of these clinical trials, several other ocular side effects have been reported, including retinal haemorrhages, and retinal vein occlusion. ${ }^{22-24}$

\section{Pathophysiology of FAME}

The proposed pathophysiological mechanism of FAME is based upon the interaction between fingolimod and $\mathrm{S}_{\mathrm{PPR}}$ present on endothelial cells in retinal vessels. S1PR signalling is responsible for maintaining cell-to-cell and cell-to-matrix adhesion complexes. The use of fingolimod is thought to downregulate this receptor, thus leading to downregulation of adhesion complexes and subsequent increased retinal vascular permeability resulting in oedema. 25,26

\section{Incidence of FAME}

Macular oedema has been a well-documented side effect of fingolimod since it was originally evaluated as an anti- 
rejection agent for renal transplantation. ${ }^{27,28}$ Fingolimodassociated macular oedema was therefore monitored for and reported in the initial clinical trials investigating the efficacy of fingolimod for RRMS. The FREEDOMS study was a phase III multicentre, 24-month, double blind randomised study comparing $0.5 \mathrm{mg}(n=425)$ and $1.25 \mathrm{mg}(n=429)$ fingolimod daily treatment with placebo ( $n=418)$ in patients with RRMS. ${ }^{1}$ None of the 425 patients receiving $0.5 \mathrm{mg}$ fingolimod developed macular oedema. Seven out of 429 (1.6\%) patients receiving $1.25 \mathrm{mg}$ fingolimod developed macular oedema and three of these were reported as serious. In 5 out of those 7 patients, macular oedema occurred within 3 months of starting treatment. In 6 out of those 7 patients, the macular oedema had resolved within 6 months of discontinuing therapy. The past ophthalmic history was not reported in any of these cases in the FREEDOMS study.

The TRANSFORMS study was a phase III multicentre, 12-month, double blind randomised study comparing fingolimod $0.5 \mathrm{mg}(n=429)$ and $1.25 \mathrm{mg}(n=420)$ to IFN $\beta$-1a intramuscularly $(n=431)$ in patients with RRMS. ${ }^{29}$ Two out of 429 patients receiving $0.5 \mathrm{mg}$ treatment $(0.5 \%)$ and 4 out of $420(1 \%)$ patients receiving $1.25 \mathrm{mg}$ treatment developed macular oedema. Three of those 6 patients were visually asymptomatic and macular oedema was diagnosed only on macular examination. Five out of those 6 patients developed macular oedema within 4 months of treatment initiation. In four out of those six patients, macular oedema had resolved within 3 months of treatment discontinuation. In the remaining two patients, one was unchanged 1 month after treatment

discontinuation and one had reduction of macular oedema 8 months after treatment discontinuation. It is not known whether these patients had ocular comorbidities prior to entering the FREEDOMS and TRANSFORMS studies. The FREEDOMS II study ${ }^{30}$ was a separate phase III clinical trial to the original FREEDOMS study and the TRANSFORMS study. It was conducted as the FDA had stipulated the need for additional monitoring, such as Holter monitoring, which was not performed in the original FREEDOMS and TRANSFORMS studies. FREEDOMS II was a phase III multicentre 24-month double-blind randomised control trial comparing fingolimod $0.5 \mathrm{mg}$ vs fingolimod $1.25 \mathrm{mg}$ vs placebo in the treatment for RRMS. Macular oedema was reported in 4 out of $370(1 \%)$ in the $1.25 \mathrm{mg}$ group, 3 out of $358(0.8 \%)$ in $0.5 \mathrm{mg}$ group and, interestingly, 2 out of $355(0.6 \%)$ in the placebo group. All those cases of FAME in this study resolved with the discontinuation of therapy, except one in the $1.25 \mathrm{mg}$ group and one in the placebo group. To our knowledge, none of the patients with resolution of macular oedema after fingolimod discontinuation were re-challenged by recommencing fingolimod in the prospective studies.
Both the FREEDOMS and TRANSFORMS studies had parallel extension studies to assess the long-term effects of fingolimod therapy. These studies found no further increased risk of FAME over a period of up to 4.5 years. ${ }^{2,31}$ In a retrospective study by Ontaneda et $a l^{32}$ a similar incidence $(3 / 317,0.9 \%)$ of macular oedema at 3 months after therapy initiation was reported.

Regarding the onset of FAME, Zarbin et al, ${ }^{33}$ using pooled analysis of the data from the phase II core and extension study with the phase III core and extension studies of FREEDOMS and TRANSFORMS, reported that FAME developed within 3-4 months of commencing fingolimod in $68 \%$ of affected cases.

From the analysis of the 15 patients (24 eyes), we found from published case reports (Table 1), the time of onset of FAME after commencement of fingolimod was within 6 months for all but two cases. In these two cases, both had been on fingolimod for an extended period ( 1 year in one case, 2 years in the other) and macular oedema only occurred after cataract surgery, suggesting that these may not be directly related to FAME per se. The majority of cases (12 out of 15) developed FAME within 4 months of initiation of therapy and 7 out of 15 occurred within 1 month. This was in keeping with the experience from the FREEDOMS and TRANSFORMS clinical trials. In summary, FAME can occur within the first 6 months of commencing fingolimod therapy. The incidence appears to be dose-dependent, occurring in $0.4 \%$ of patients treated with $0.5 \mathrm{mg}$, and in $1 \%$ of those treated with $1.25 \mathrm{mg} .{ }^{10-21}$

\section{Symptoms of FAME}

Although the cases identified in the FREEDOMS and TRANSFORMS trials were not reported in sufficient detail to review their symptomatology, the case reports were a very useful source of this information. Of the 15 patients (24 eyes) with FAME reported as case reports in the literature (Table 1), the majority were symptomatic; the most common presenting complaint being painless blurred vision. One patient had metamorphopsia and another patient was asymptomatic but actually had reduced vision of $6 / 18$. Presenting vision was mildly reduced (20/30 or better) in 8 of 24 eyes and moderately reduced (20/30-20/80) in the remainder (16 of 24 eyes). The worst visual acuity reported in an eye with FAME was 6/24 or, approximately, 20/80.

\section{Diagnosis of FAME}

In all of the 15 cases of retinal problems thought to be attributed to fingolimod therapy, all of them had abnormal signs on OCT scanning or fluorescein angiography of either macular thickening, foveal or 


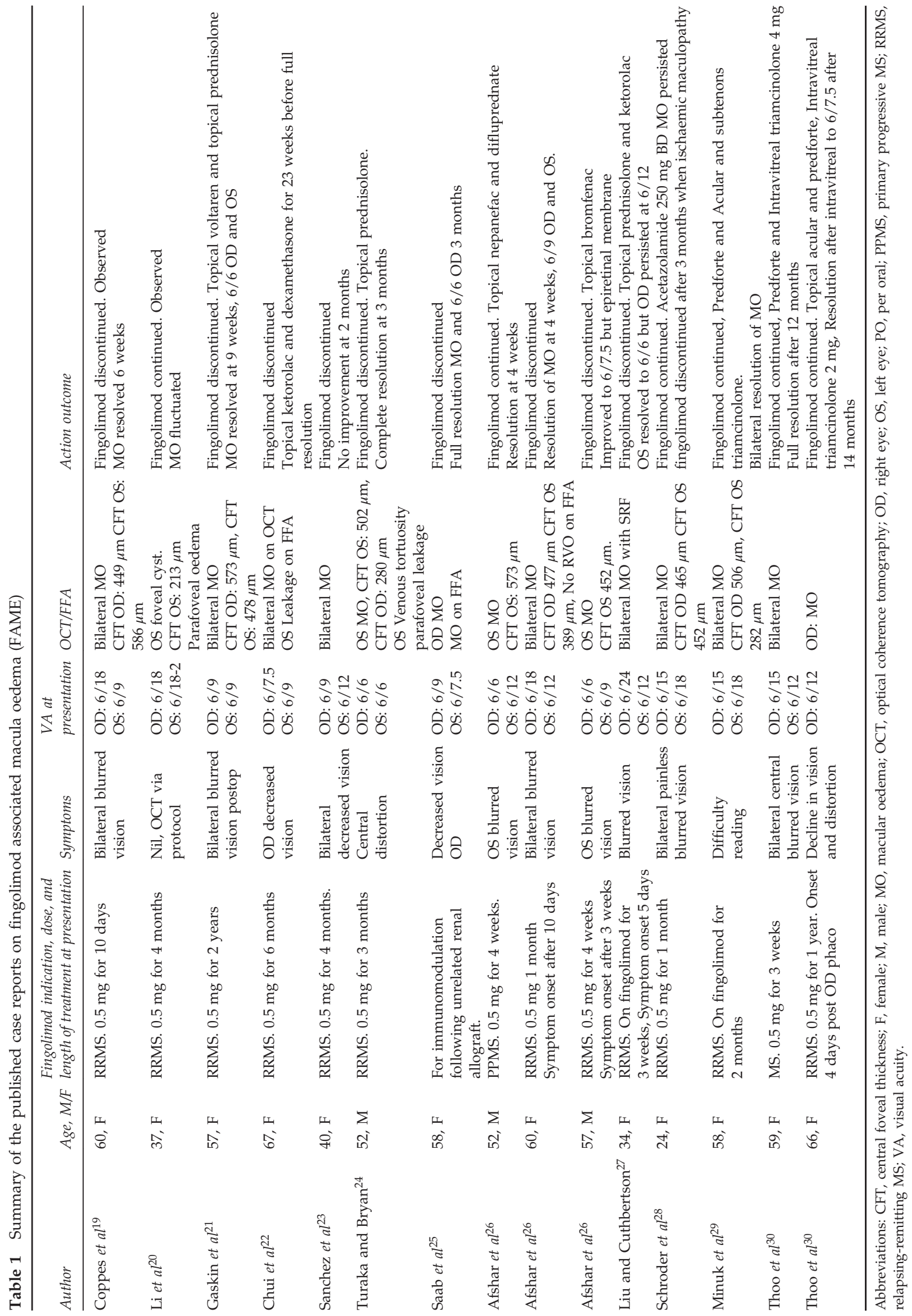


perifoveal cysts, subretinal fluid, venous tortuosity or dye leakage. These findings are easily visible on biomicroscopy and OCT scanning. Fluorescein angiography can be used to rule out other causes of macular oedema such as posterior uveitis or retinal vein occlusion, or diabetic retinopathy. In making the diagnosis of FAME, it is important to be aware that neuronal loss and retinal thinning can result in the presence of degenerative microcysts in about $4-5 \%$ of patients with MS, typically seen in the inner nuclear layer on OCT scanning. Although these cysts have been termed microcystic macular oedema secondary to multiple sclerosis, they are probably not due to transudation or exudation, as they are not known to be associated with reduced visual acuity or dye leakage on fluorescein angiography and are more often associated with reduced macular volume and overall retinal thinning, particularly in patients with long-standing or severe MS. ${ }^{34,35}$

\section{Management of FAME}

Although Zarbin et $a l^{33}$ reported that $84 \%$ of patients had macular oedema resolution after fingolimod cessation in the All Studies group, there are numerous reports of cases in which discontinuation did not lead to resolution and needed topical prednisolone and ketorolac. ${ }^{18}$ There are also reports on cases in which fingolimod was not discontinued and this led to persistence of $\mathrm{MO},{ }^{11}$ and variable resolution with topical nepafenac and difluprednate, ${ }^{17}$ topical ketorolac and dexamethasone, ${ }^{13}$ oral acetazolamide, ${ }^{19}$ sub-tenon triamcinolone, ${ }^{20}$ and intravitreal triamcinolone. ${ }^{21}$ From these reports, it appears that the current steps for managing FAME is to firstly confirm the diagnosis with OCT scan and fluorescein angiography to rule out other causes of MO and then discontinue fingolimod, if possible. Persistent $\mathrm{MO}$ on early follow-up can then be treated with topical steroidal or non-steroidal agents initially before introducing subtenon or intravitreal triamcinolone. There is no evidence base available at present for the use of intravitreal ozurdex, intravitreal anti-VEGF, or oral corticosteroids in the management of FAME.

\section{FAME and its association with uveitis}

Zarbin et $a^{33}$ suggested a higher risk of developing FAME in patients with a history of uveitis than those without. Using pooled analysis of the data from the phase II core and extension study with the phase III core and extension studies of FREEDOMS and TRANSFORMS $(\mathrm{N}=2615)$, a total of 19 cases had reported macular oedema. The prevalence of patients with a history of uveitis in this dataset was 1\% (26 out of 2615), but interestingly amongst those who developed macular oedema, the prevalence of a history of uveitis was $26 \%$ (5 out of 19 ). Thus, the incidence of macular oedema amongst those with a history of uveitis was $19 \%(5 / 26)$ compared with the overall incidence of macular oedema in the dataset being $0.7 \%$ (19 out of 2615). All five patients with uveitis who developed macular oedema were taking $1.25 \mathrm{mg}$ fingolimod. It is difficult to comment on the link between FAME and uveitis from the case reports detailed in Table 1, as there is no control for comparison, but it can be noted that out of the 14 case reports containing sufficient detail on past ocular history, there were only two patients with a history of uveitis prior to commencing fingolimod. The findings from the analysis of these anecdotal case reports therefore support the suggestion by Zarbin et al that, although patients without any history of uveitis can develop FAME, those with a past history of uveitis probably have an increased risk of developing FAME.

\section{FAME and its association with diabetes}

Patients with diabetes mellitus were excluded from the FREEDOMS and TRANSFORMS clinical trials. The clinical trials assessing the use of fingolimod as an antirejection agent in renal transplant patients was reported by Salvadori et $a l^{27}$ and Tedesco-Silva et al. ${ }^{28}$ Both these clinical trials evaluated higher-dose fingolimod (5 and $2.5 \mathrm{mg}$ ) in association with cyclosporine, and both reported higher rates of macular oedema (Salvadori et al: $2.2 \%$ at $5 \mathrm{mg}$ dose, $1.3 \%$ at $2.5 \mathrm{mg}$ dose), (Tedesco-Silva et al: $3.4 \%$ at $5 \mathrm{mg}$ dose and $1.7 \%$ at $2.5 \mathrm{mg}$ ). Tedesco-Silva and Salvadori did not exclude patients with diabetes in the sample population. However, as doses used were up to 10 times higher than the licensed dose today, it is difficult to state whether this increase in rate of FAME is due to the higher dosage or the fact that diabetic patients were included in the sample. In addition, as diabetic patients were not excluded from the study, it is difficult to conclude whether the macular oedema was caused by fingolimod or related to diabetic maculopathy.

Furthermore, in the case reports we reviewed, only 2 out of 14 patients had diabetes and one with no diabetic retinopathy, there is insufficient evidence to suggest that patients with diabetes or diabetic retinopathy without maculopathy are at an increased risk of developing FAME with fingolimod.

It is reasonable, however, to suspect that diabetic patients are more likely to be prone to macular oedema than non-diabetic patients, due to an already compromised blood-retinal barrier, thus, fingolimod must be used with caution in this cohort of patients. 


\section{Fingolimod and retinal haemorrhages}

To date, there have been two cases of retinal haemorrhage in patients treated with fingolimod therapy. Bhatti et al ${ }^{22}$ report a 54-year-old female, with no history of diabetes mellitus or hypertension, who had been treated with $0.5 \mathrm{mg}$ of fingolimod for 11 months for RRMS. She presented with a grey opaque spot in her visual field and VA of 20/80. A unilateral, dense retinal haemorrhage involving the fovea with an adjacent hard exudate and macular thickening were confirmed on OCT in her left eye. The FFA of the affected eye revealed fluorescein blockage due to blood, and lipid exudates and hyperfluorescence adjacent due to areas of blockage, but without overt angiographic signs of retinal vein occlusion. Fingolimod was promptly discontinued and 1 month later the macular haemorrhage had completely resolved. By 3 months, visual acuity had recovered to baseline of 20/30 and OCT was normal. Ueda and Saida reported a case of a 31-year-old male with RRMS and pre-existing poor visual acuity (OD 20/600, OS 20/400), who developed both macular oedema and retinal haemorrhages in all four quadrants unilaterally in his left eye 1 month after commencing fingolimod therapy. Treatment was discontinued, but due to persisting FAME 13 weeks after cessation of therapy, topical betamethasone $0.1 \%$ was commenced. The macular oedema resolved 4 weeks after this addition of topical steroid (17 weeks after cessation of fingolimod), and the haemorrhages resolved 11 weeks after the addition of topical steroid ( 24 weeks after cessation of fingolimod). ${ }^{23}$ The mechanism by which fingolimod may cause retinal haemorrhages has not yet been fully elucidated, though it may also be linked with the $\mathrm{S}_{\mathrm{PR}}$ mechanism of FAME, rendering retinal blood vessels increasingly permeable. $^{25,26}$

\section{Fingolimod and its association with retinal vein occlusion}

As retinal vein occlusion (RVO) is a common condition, it is uncertain whether a link exists between RVO and fingolimod therapy. To date, only one previous report of branch retinal vein occlusion has been published in the literature. Gallego-Pinazo et $a^{24}$ described a case of a 47-year-old female patient with a 9-year history of MS. She had been treated with fingolimod for 6 years prior to developing unilateral sudden visual reduction in her left eye to 20/40 due to a superotemporal BRVO associated with macular oedema and central foveal thickness of $396 \mu \mathrm{m}$. FFA typically revealed an area of delayed venous filling and blockage of fluorescence by the intraretinal haemorrhages. Fingolimod was discontinued and the patient was treated with one intravitreal ranibizumab injection. The patient had neither cardiovascular risk factors, nor any coagulation abnormality, suggestive of an alternative cause for RVO. Three weeks later, visual acuity had improved to 20/20 in the patient's left eye and there was almost complete resolution of retinal oedema. It is uncertain whether fingolimod has a thrombogenic effect in veins but Nealon et $a l^{36}$ describe a case of a patient who developed a thrombus in a developmental venous angioma 2 months after commencing fingolimod therapy. Similar to the patient described by GallegoPinazo, this patient also did not have any recognised cardiovascular risk factors or coagulation abnormality. Thus, the authors concluded that fingolimod could be thrombogenic. Schwarz et $a l^{37}$ reported a patient who developed critical arterial vasospasm of the left arm within 7 days of commencing fingolimod therapy. Considering the half-life of fingolimod is 9 days and the maximum vasospasm occurred at 13 days postdiscontinuation, they argued that this chronological sequence is suggestive of a causal relationship between fingolimod and vasospasm. If vasospasm were to occur at the point of an AV crossing, a retinal vein occlusion would ensue. Lastly, as fingolimod causes a rise in blood pressure, one could argue that this could have facilitated retinal vein occlusion. However, due to the transient and also rather modest (+2 $\mathrm{mm} \mathrm{Hg}$ systolic) rise in blood pressure, this mechanism seems unlikely. 6,7

\section{Ophthalmic monitoring in patients on fingolimod therapy}

The time of onset of FAME after commencement of fingolimod was within 6 months for all but two cases. Both of these cases had been on fingolimod for an extended period ( 1 year in one case, 2 years in the other) and $\mathrm{CMO}$ developed in both these cases only after cataract surgery, a known trigger for developing CMO. The majority of cases (12/15) developed FAME within 4 months of initiation of therapy, keeping in line with the original FREEDOMS and TRANSFORMS clinical trials. Therefore, screening patients on fingolimod after 3-4 months as per NICE guidelines seems appropriate. Moosavi et $a l^{38}$ performed a retrospective study to assess the adherence to these NICE guidelines and to measure the impact of delivery of these guidelines on clinical service. In a 9-month period, 38 referrals for fingolimod screening were made, contributing to a significant $9 \%$ of new referrals. Only 1 patient had FAME, who had in fact developed blurred vision soon after fingolimod initiation and was seen in the eye clinic at 5 weeks post commencement where OCT confirmed FAME; shorter than the 3-4 month recommendation. Moosavi et al concluded that reviewing fingolimod patients at $3-4$ months is a huge burden to eye services for a rare 
side effect. They recommend that near and distance acuity is measured by the physician prescribing fingolimod, and referrals to an ophthalmologist should only be made if there is a reduction in acuity or a change in visual symptoms. As with Moosavi's study, we also found from most of the case reports published that the majority of

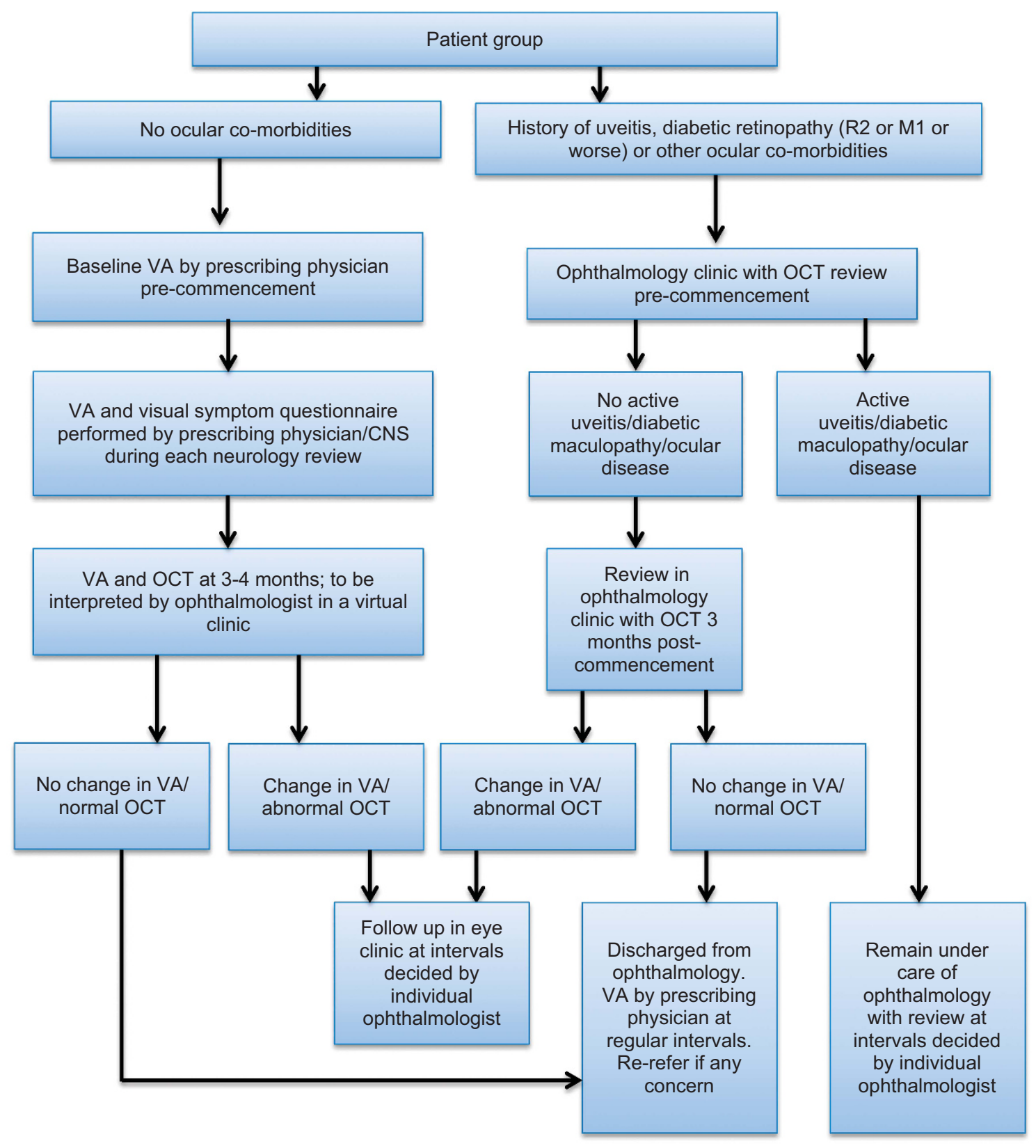

All patients on fingolimod undergoing intraocular surgery to have eye clinic review with OCT preoperatively and at 1 month postoperatively

Legend: VA- Visual acuity, OCT-Optical Coherence Tomography

Figure 1 Proposed pathway for screening of patients on fingolimod therapy. All patients on fingolimod undergoing intraocular surgery to have eye clinic review with OCT preoperatively and at 1 month postoperatively. 
patients had symptoms of visual blurring. This is in contrast to the original FREEDOMS and TRANSFORMS trials that reported fingolimod ocular effects to be asymptomatic. This perception from the FREEDOMS and TRANSFORMS trials that fingolimod effects on the eye is often asymptomatic may be the reason for the stipulation by NICE for routine ophthalmic screening at 3-4 months in all patients commencing fingolimod therapy. Given the low incidence of ocular effects and the higher likelihood that such effects are associated with visual symptoms, we would like to propose a pragmatic pathway for implementing the NICE guidelines on screening. Figure 1 shows the proposed pathway based on previous ocular history and a simple visual acuity measurement prior to discontinuation of fingolimod or referral to hospital ophthalmology departments. If there is a past history of uveitis or diabetic retinopathy, patients could be screened before commencement of fingolimod to rule out active maculopathy, as the addition of fingolimod may exacerbate pre-existing disease. In those patients with a history of ocular comorbidities but no active disease, review in eye clinic within the most likely time window for the development of FAME (3-4 months) is suggested. However, in those with no significant ophthalmic history, we propose that a baseline visual acuity measurement is performed by the physician prescribing fingolimod followed by a further visual acuity test and OCT 3-4 months later in a virtual eye clinic. In the unlikely event that a patient with FAME is asymptomatic, there will still be an opportunity for such a patient to be detected in this screening pathway at the second contact in the virtual eye clinic. As the risk of FAME is low after 4 months, screening beyond this time is probably unnecessary. However, as patients on fingolimod therapy, regardless of duration, who undergo intraocular surgery are at an increased risk of FAME, it is suggested that this cohort of patients have a routine OCT at the preand postoperative check.

\section{Conclusion}

In summary, there is a significant association of macular oedema (FAME) and other retinal vascular problems in patients who are on treatment with fingolimod for MS. Fortunately, the visual consequences appear to be mild and often resolve on discontinuation of fingolimod therapy. The pathophysiologic mechanism responsible for FAME is thought to be due to an increased permeability of the retinal vessels due to the effect of fingolimod on retinal sphingolipid receptors. However, in rarer cases where there is retinal vein occlusion, discontinuation of fingolimod alone may not lead to resolution and intravitreal therapy may be required. Due to its efficacy in treating multiple sclerosis symptoms, fingolimod may become more widely used. Thus, we suggest that ophthalmic monitoring of patients should be based upon their visual acuity, The OCT findings and comorbidities enabling close monitoring of those at-risk while ensuring the burden is manageable. Continued reporting of other cases in the scientific literature and via the yellow card system should further our knowledge and experience of this new cause of macular oedema.

\section{Conflict of interest}

The authors declare no conflict of interest.

\section{References}

1 Kappos L, Radue E-W, O'Connor P, Polman C, Hohlfeld R, Calabresi $\mathrm{P}$ et al. A placebo-controlled trial of oral fingolimod in relapsing multiple sclerosis. N Engl J Med 2010; 362(5): 387-401.

2 Kappos L, O'Connor P, Radue EW, Polman C, Hohlfeld R, Selmaj $\mathrm{K}$ et al. Long-term effects of fingolimod in multiple sclerosis: the randomized FREEDOMS extension trial. Neurology 2015; 84(15): 1582-1591.

3 Groves A, Kihara Y, Chun J. Fingolimod: direct CNS effects on sphingosine 1-phosphate (S1) receptor modulation and implications in multiple sclerosis therapy. J Neurol Sci 2013; 328(1-2): 9-18.

4 Chun J, Hartung H. Mechanism of action of oral fingolimod (FTY720) in multiple sclerosis. Clin Neuropharmacol 2010; 33(2): 91-101.

5 NICE guidelines Fingolimod, SPC fingolimod joint Formulary Committee. British National Formulary. 70th ed. BMJ Group and Pharmaceutical Press: London, UK, 2015.

6 Aguiar C, Batista S, Pachero R. Cardiovascular effects of fingolimod: relevance, detection and approach. Rev Port Cardiol 2015; 34(4): 279-285.

7 Camm J, Hla T, Bakshi R, Brinkmann V. Cardiac and vascular effects of fingolimod: Mechanistic basis and clinical implications. Am Heart J 2014; 168(5): 632-644.

8 Brush RS, Tran J, Henry K, mcclellan M, McClellan ME, Elliott $\mathrm{MH}$ et al. Retinal sphingolipids and their very-longchain fatty acid-containing species. Invest Ophthalmol Vis Sci 2010; 51(9): 4422-4431.

9 Chen H, Chan AY, Stone DU, Mandal NA. Beyond the cherry-red spot: ocular manifestations of sphingolipidmediated neurodegenerative and inflammatory disorders. Surv Ophthlamol 2014; 59(1): 64-76.

10 Coppes OJ, Gutierrez I, Reder AT, Ksiazek S, Bernard J. Severe early bilateral macular edema following fingolimod therapy. Mult Scler Relat Disord 2013; 2(3): 256-258.

11 Li V, Kane J, Chan HH, Hall AJ, Butzkueven H. Continuing fingolimod after development of macular edema: a case report. Neurol Neuroimmunol Neuroinflamm 2014; 1(2): e13.

12 Gaskin JC, Coote M. Postoperative cystoid macular oedema in a patient on fingolimod. BMJ Case Rep; e-pub ahead of print 12 May 2015; doi:10.1136/bcr-2015-210415.

13 Chui J, Herkes GK, Chang A. Management of fingolimodassociated macular edema. JAMA Ophthalmol 2013; 131(5): 694-696.

14 Asensio-Sanchez VM, Trujillo-Guzman L, Ramoa-Osorio R. Cystoid macular oedema after fingolimod treatment in multiple sclerosis. Arch Soc Esp Oftalmol 2014; 89(3): 104-106. 
15 Turaka K, Bryan JS. Does fingolimod in multiple sclerosis patients cause macule edema? J Neurol 2012; 259(2): 386-388.

16 Saab G, Almony A, Blinder KJ, Schuessler R, Brennan DC. Reversible cystoid macular edema secondary to fingolimod in a renal transplant recipient. Arch Ophthalmol 2008; 126(1): 140-141.

17 Afshar AR, Fernandes JK, Patel RD, Ksiazek SM, Sheth VR, Reder AT et al. Cystoid macular edema associated with fingolimod use for multiple sclerosis. JAMA Ophthalmol 2013; 131(1): 103-107.

18 Liu L, Cuthbertson F. Early Bilateral cystoid macular oedema secondary to fingolimod in multiple sclerosis. Case Rep Med 2012; 2012: 134636.

19 Schroder K, Finis D, Harmel J, Ringelstein M, Hartung HP, Geerling $\mathrm{G}$ et al. Acetazolamide therapy in a case of fingolimod-associated macular edema: early benefits and long-term limitations. Mult Scler Relat Disord 2015; 4(5): 406-408.

20 Minuk A, Belliveau MJ, Almeida DR, Dorrepaal SJ, Gale JS. Fingolimod-associated macular edema: resolution by sub-tenon injection of triamcinolone with continued fingolimod use. JAMA ophthalmol 2013; 131(6): 802-804.

21 Thoo S, Cugati S, Lee A, Chen C. Successful treatment of fingolimod-associated macular edema with intravitreal triamcinolone with continued fingolimod use. Mult Scler 2015; 21(2): 249-251.

22 Bhatti MT, Freedman SM, Mahmoud TH. Fingolimod therapy and macular haemorrhage. J Neuroophthalmol 2013; 33(4): 370-372.

23 Ueda N, Saida K. Retinal haemorrhages following fingolimod treatment for multiple sclerosis; a case report. BMC Ophthalmol 2015; 15(1): 135.

24 Gallego-Pinazo R, España-Gregori E, Casanova B, Pardo-López D, Diaz-Llopis M. Branch retinal vein occlusion during fingolimod treatment in a patient with multiple sclerosis. J Neuroophthalmol 2011; 31(3): 292-293.

25 McVerry BJ, Garcia JG. Endothelial cell barrier regulation by sphingosine 1-phosphate. J Cell Biochem 2004; 92(6): 1075-1085.

26 Oo ML, Chang SH, Thangada S, Wu MT, Rezaul K, Blaho V et al. Engagement of S1P(1)-degradative mechanisms leads to vascular leak in mice. J Clin Invest 2011; 121(6): 2290-2300.

27 Salvadori M, Budde K, Charpentier B, Klempnauer J, Nashan B, Pallardo LM et al. FTY720 versus MMF with cyclosporine in de novo renal transplantation: a 1-year, randomized controlled trial in Europe and Australasia. Am J Transplant 2006; 6(12): 2912-2921.

28 Tedesco-Silva H, Pescovitz MD, Cibrik D, Rees MA, Mulgaonkar, Kahan BD et al. Randomized controlled trial of FTY720 versus MMF in de novo renal transplantation. Transplantation 2006; 82: 1689-1697.

29 Cohen JA, Barkhof F, Comi G, Hartung H-P, Khatri BO, Montalban $\mathrm{X}$ et al. Oral fingolimod or intramuscular interferon for relapsing multiple sclerosis. N Engl J Med 2010; 362(5): 402-415.

30 Calabresi PA, Radue EW, Goodin D, Jeffery D, Rammohan KW, Reder AT et al. Safety and efficacy of fingolimod in patients with relapsing-remitting multiple sclerosis (FREEDOMS II): a double-blind, randomised, placebo-controlled, phase 3 trial. Lancet Neurol 2014; 13(6): 545-556.

31 Cohen JA, Khatri B, Barkhof F, Comi G, Hartung HP, Montalban $\mathrm{X}$ et al. Long-term (up to 4.5 years) treatment with fingolimod in multiple sclerosis: results from the extension of the randomised TRANSFORMS study. J Neurol Neurosurg Psychiatry 2015; 87(5): 468-475.

32 Ontaneda D, Hara-Cleaver C, Rudick RA, Cohen JA, Bermel RA. Early tolerability and safety of fingolimod in clinical practice. J Neurol Sci 2012; 323(1-2): 167-172.

33 Zarbin MA, Jampol LM, Jager RD, Reder AT, Francis G, Collins W et al. Ophthalmic evaluations in clinical studies of fingolimod (FTY720) in multiple sclerosis. Ophthalmology 2013; 120(7): 1432-1439.

34 Brar M, Yuson R, Kozak I, Mojana F, Cheng L, Bartsch DU et al. Correlation between morphologic features on spectraldomain optical coherence tomography and angiographic leakage patterns in macular edema. Retina 2010; 30: 383-389.

35 Gelfand JM, Nolan R, Schwartz DM, Graves J, Green AJ. Microcystic macular oedema in multiple sclerosis is associated with disease severity. Brain 2012; 135(6): 1786-1793.

36 Nealon B, Ternopolska N, White H. Report an Usual Thrombotic Complication Two Months After Starting Fingolimod. Neurology Supplement 2014; 82(10): 2.215.

37 Schwarz A, Korporal M, Hosch W, Max R, Wildemann B. Critical vasospasm during fingolimod (FTY720) treatment in a patient with multiple sclerosis. Neurology 2010; 74(24): 2022-2024.

38 Moosavi R, Bremner F, Acheson J. Letter to the editor screening for fingolimod associated macular oedema: experience versus. Open Ophthalmol J 2014; 8: 73-74. 